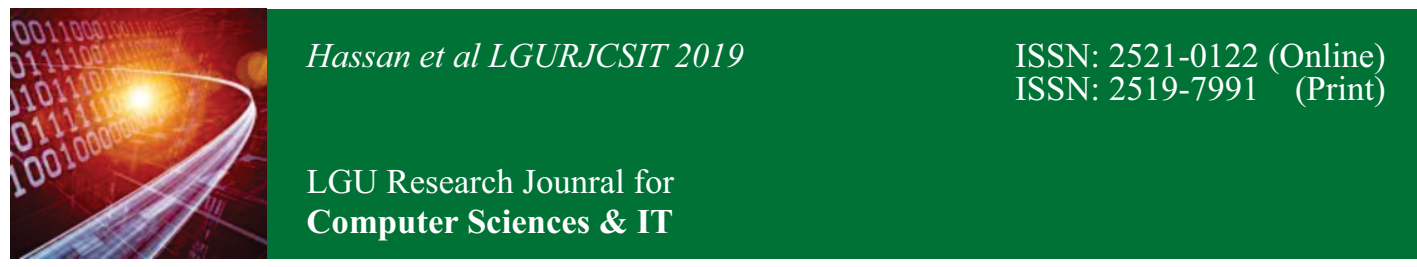

Vol. 3 Issue 4, October - December 2019

\title{
Software Testing for cyber Security
}

\author{
Arfa Hassan, Tayyaba Anees, Adnan Khan
}

\begin{abstract}
:
Software testing plays a vital role in software security because hackers attack on a system through back channels which they can easily find if there is any error or bug exists in the software. The software security failure can cause the unbearable loss for IT companies and other organizations. Cyber security is another big issue for computer users' personal data as all their information is vulnerable because of easy excess, visibility and availability. Therefore, software testing is also useful to secure the personal information. In this article, cyber security testing based on particle swam optimization algorithm (CST) is proposed for testing of software cyber security testing. CSTPSOA is a PSO base technique which is used to solve the complex multi-level problems and is also used for optimization. In the CST method PSO is used for the optimization of test cases for cyber security testing.
\end{abstract}

Keywords: $\quad$ Cyber Security, CST, Software Testing, PSO, Quality Assurance

\section{Introduction}

Software testing is the process of software quality assurance which is used to figure out the faults, defects, errors and bugs of the developing and as well as the underdeveloped software.[1] Experts divide the software testing phases into three categories i.e., functional testing, nonfunctional testing and regression testing. Software testing is constructive to reduce the maintenance cost. And it is also used to determine the security risks of the software before its deployment. The security of any software is an important feature because majority of activities of today's human is controlled through software[2]. And thus software security barrier can cause the unbearable loss. An ACI (army cyber institute) report shows that innovation in the cloud computation and IOT devices make a global shortage of cyber-security talent in 2017. This gap between cyber security talent and white collar criminals can create the political, personal and business consequences such as panama leaks and wiki leaks. [3]The reason behind these cyber security attacks are that companies show less interest towards cyber security as sometimes the authorities of these companies have not enough awareness regarding white collar crime and also another major issue is that the different vendors of the devices follows different kind of rules for the cyber security[4].

The most recent incident in this context occurs with Careem ride service in Pakistan. The hackers hack their website and steel all the important information of their customer. The stolen data also includes some personal information like their credit card details etc. The main focus of hackers in such incidents are to steel the banking details, phone numbers and other personal details which they can further use for illegal activities [5].

The process of identifying, analysis and evaluation of software before the implementation of that software is important for its protection and unauthorized handling. The results of the survey 2017 on cyber security breaches arranged by the UK government reveals that main cause of cyber-attacks is that the companies do not care about the cyber security at foundational levels because they think that they are not at risk. Cyber security risk assessment is equally important for the hardware because many hackers also able to access the 
hardware component such as Ram, Rom and attached device, for example, USB, external hard drive, printer etc. [5].

Test case generation and evolution is an important task for the SQA professional. This research article CST method is proposed for software testing. CST algorithm used Particle swarm optimization technique for cyber security testing test case optimization. PSO is a greedy approach of artificial intelligent to get best results in less time[6].

\section{Literature Reviews}

Researches introduce different kinds of algorithm for the software testing and software security testing. A few of them are discussed below.

PSO based greedy algorithm approach is very useful in regression testing. Regression testing looks expensive, but it is really helpful to reduce the maintenance cost of the software. For this purpose the researchers takes the sample set of 5 different softwares and test them line by line through the sample test set[7].

Web base application is main source of business, communication and information sharing so protection of web based application from hackers attack is necessary. Software security testing before implementation of such type of applications can play important role. In [8] article the researcher used the genetic algorithm based approach for web based applications security testing. This proposed method could be able to perform analysis of expected breaches and divide the problem in two categories after analysis.

$\begin{array}{ll}- & \text { Static analysis } \\ \text { - } & \text { Dynamic analysis }\end{array}$

This analysis of the software creates the fitness function and check the security issues of the software.

Automatic software testing tools can play vital role in software quality assurance. Software quality assurance helps to reduce about $50 \%$ of software development cost[9]. The proposed algorithm of this purpose is feed forward neural network with back propagation learning algorithm. Two models are used in this approach. Model 1 takes 2 input variables but in model 2 researchers uses 4 variables[9]. In another article, the researchers work on fault detection techniques. For this purpose, they apply Rank-to-learn algorithm. To enhance the performance of rank-to-learn technique they also use the back propagation. To evaluate this system they take 10 different cases. For the simulation matlab is used[10].

The basic aim of today's software industry is to provide high quality secure software to the end users. Software testing techniques can play a vital role in the software quality assurance. The field of software testing have various issues which require efforts on time and cost of testing. For this purpose the researcher conduct the survey on Genetic algorithm techniques. In this article number of techniques are discussed which use genetic algorithm techniques for software testing tools development[11].

\section{Proposed Method}

In this article CST method is proposed for software security testing. CST works on the Particle swam optimization. PSO is stochastic optimization technique[12]. PSO can be applied on various problems of different fields. PSO is a population based computational problem solving method[7]. The basic idea of PSO is taken form the flak of swarms[13]. The step of PSO is as following:

- $\quad$ Initial the population
- $\quad$ Fvaluate the fitness
- $\quad$ Find the Garticle best value
$\quad$ Create the stopping condition.

The mathematical formulation of particle best and global best values is given below:

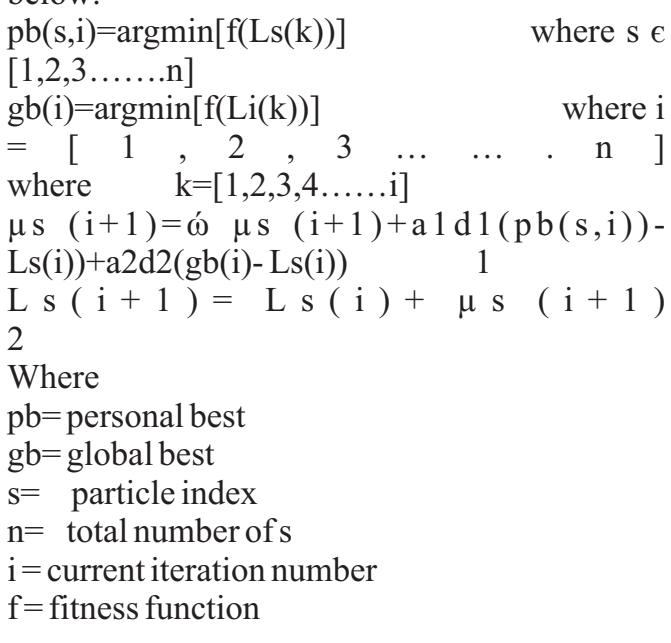


$\mathrm{L}=$ position

$\mu=$ velocity

$\dot{\omega}=$ weight

$\mathrm{d} 1 \& \mathrm{~d} 2=$ random variables

a1 \& a2= acceleration coefficients.

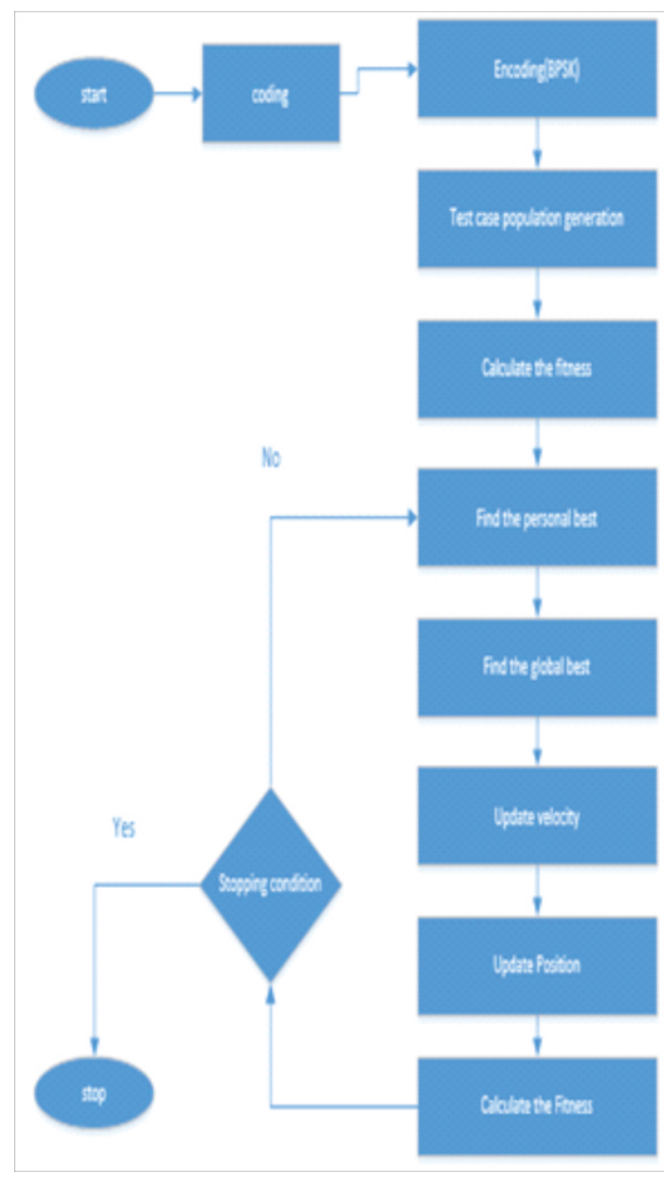

Figure 1: Flow chart of CST

Software security is a sensitive issue so many different techniques are developed by the SQA professionals for software testing. Formal method [14] and model based techniques are one them. NASA[2] introduced formal methods for software testing. Formal method is a way of software testing in which whole software is converted into pieces of some meaningful algorithm[15]. In the model based software testing approach software tests are designed through the UML [16] diagrams of software and generate test case through the standard software[17]. The proposed method of this article is CST (cyber security testing) based on particle swarm optimization algorithm (PSO). For this purpose the first step is to convert the whole software in a binary form, then encode it to remove 0 values form the code. After initialization of the test case population, optimization process is start. For this purpose algorithm evaluate the fitness function and then finds the local best or global best value. If the global best value are between the fitness functions value or required number of iterations are complete then stop this process. The complete process of proposed CST method is shown in figure 1.

\section{Simulation and results}

For simulation and results Matlab R2017a tool is used. For simulation and results Matlab R2017a tool is used. The proposed system further illustration through an example. Table 1 shows the population and table 2 shows the fitness test.

\begin{tabular}{|l|l|}
\hline $\begin{array}{l}\text { Sr } \\
\text { no. }\end{array}$ & Population \\
\hline 1 & $1,1,-1,-1,-1,-1,1,-1,1,-1,1,-1,1,-1,1,-1,1,-1,1,-1,1,1$ \\
\hline 2 & $-1,1,1,-1,1,-1,1,-1,1,-1,1,1,-1,1,-1,1,-1,-1,1,-1,1,1$ \\
\hline 3 & $1,1,1,1,1,1,1,1,1,1,-1,1,-1,1,-1,1,-1,1,-1,1,-1,1$ \\
\hline 4 & $-1,1,-1,1,-1,1,-1,1,-1,1,-1,1,-1,1,-1,1,1,1,-1,-1,-1,1$ \\
\hline 5 & $-1,1,-1,1,-1,1,-1,1,-1,-1,1,1,1,1,-1,-1,1,-1,1,-1,1,-1$ \\
\hline 6 & $1,-1,1,-1,1,-1,1,-1,1,-1,1,-1,1,-1,1,-1,1,-1,1,-1,1,-1$ \\
\hline 7 & $-1,1,-1,1,-1,1,-1,1,-1,1,-1,1,-1,1,-1,1,-1,1,-1,1,-1,1$ \\
\hline 8 & $1,-1,1,-1,1,-1,1,-1,1,1,-1,-1,1,-1,1,-1,1,1,-1,1,-1,1$ \\
\hline 9 & $-1,1,-1,-1,-1,1,-1,1,-1,1,-1,1,-1,-1,-1,1,-1,1,-1,1,-1,1$ \\
\hline 10 & $1,-1,1,-1,1,-1,1,-1,-1,-1,1,-1,1,-1,1,-1,1,1,-1,-1,-1,-1$ \\
\hline 11 & $-1,1,-1,1,-1,1,-1,1,-1,1,-1,1,-1,-1,-1,-1,-1,-1,1,1,1,1$ \\
\hline 12 & $-1,1,-1,1,-1,1,-1,-1,-1,-1,1,-1,1,-1,1,-1,1,1,-1,1,-1,-1$ \\
\hline 13 & $-1,1,-1,1,1,1,-1,-1,-1,-1,1,1,1,1,-1,-1,1,-1,1,-1,-1,1$ \\
\hline 14 & $-1,-1,-1,1,1,1,1,-1,-1,1,-1,1,-1,1,-1,1,1,-1,-1,1,1,-1$ \\
\hline 15 & $-1,-1,-1,1,1,-1,1,-1,1,-1,1,-1,1,-1,1,-1,1,1,-1,-1,1,-1$ \\
\hline
\end{tabular}

Table 1: BPSK Test case population initialization.

\begin{tabular}{|l|l|}
\hline Sr no. & Fitness function \\
\hline 1 & $-1,1,-1,1,-1,1,-1,1,-1,1,1,-1,-1,1,1,-1,-1,1,1,-1,-1$ \\
\hline 2 & $-1,1,-1,1,-1,-1,1,-1,1,-1,1,-1,1,-1,1,-1,1,-1,1,-1,1$ \\
\hline
\end{tabular}

Table 2: BPSK Fitness function The cost function of proposed CST based pso method is

(Gbesti/Pbesti).xi 
Figure 2 shows the matlab graph results of proposed PSO based CST system.

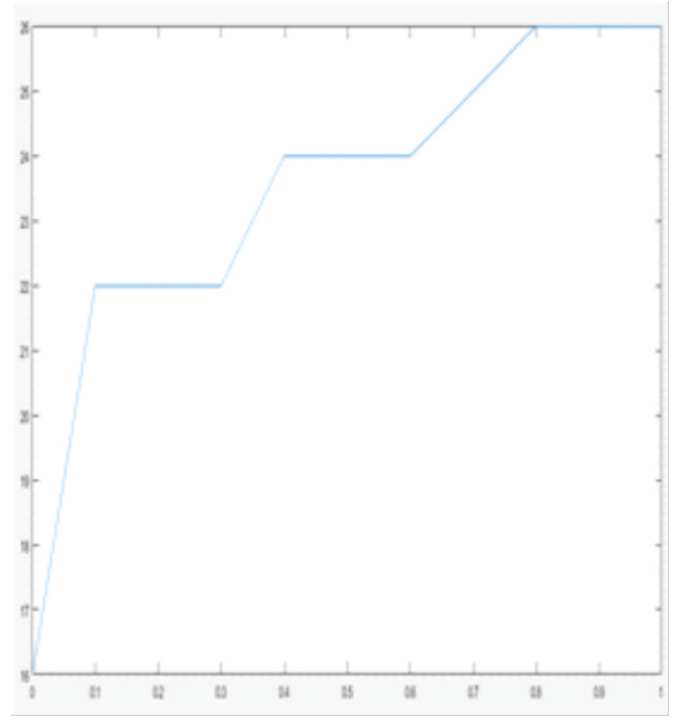

Figure 2.PSO with different number of cycles

\section{Conculsion}

Cyber security is a sensitive issue and required great attention. But actually this is the most neglected part of SDLC especially in under developed countries like Pakistan. Campiness can easily secure their products by performing proper software security testing on their products. Test case generation is major and the most challenging part of software testing process. Sometime thousands of test cases are generated by the software, which makes the software testing job more tough and complicated. In this article CST algorithm is proposed for test case optimization. The proposed method uses the PSO algorithm for optimization of test cases. The proposed method could also be used to find out the perfect test cases on the basis of fitness test.

\section{REFERENCES}

[1] J. J. Scarpino, "Web Application Security Testing : an Industry Perspective on How Its Education Is Perceived," vol. XI, no. 1, pp. 142-153, 2010.

[2] Gu Tian-yang, Shi Yin-sheng, and Fang You-yuan, "Research on Software Security Testing," Int. J. Comput. Electr. Autom. Control Inf. Eng., vol. 4, no. 9, pp. 1447-1450, 2010.
[3] A. Bendovschi, "Cyber-Attacks Trends, Patterns and Security Countermeasures," Procedia Econ. Financ., vol. 28, no. April, pp. 24-31, 2015.

[4] J. Twist, "ACI Threat Trends and Predictions 2017 Report,” 2017.

[5] Becky Metivier, "6 Steps to a Cybersecurity Risk Assessment," sage data security, 2017. [Online]. Available: https://www.sagedatasecurity.com/blog/6steps-to-a-cybersecurity-risk-assessment. [Accessed: 26-Mar-2018].

[6] D. Palupi Rini, S. Mariyam Shamsuddin, and S. Sophiyati Yuhaniz, "Particle Swarm Optimization: Technique, System and Challenges," Int. J. Comput. Appl., vol. 14, no. 1,pp. 19-27, 2011.

[7] U. Jafri, H. Sadia, and J. Ahmad, "PSO based Optimized Software Testing Technique," vol. 13, no. 2, 2017.

[8] A. Avancini and F. B. Kessler, "Security Testing of Web Applications : A Research Plan," no. line 1, pp. 1491-1494, 2012.

[9] Q. P. Rana, "Model for Software Testing and Quality Assessment using ANN Approach," vol. 14, no. 1, 2017.

[10] P. K. Sidhu, "Evaluate and Propose Fault Detection Technique from Test Cases in Software Testing,"vol. 5, no. 3, pp.9-14, 2017.

[11] C. Sharma, S. Sabharwal, and R. Sibal, "A Survey on Software Testing Techniques using Genetic Algorithm," vol. 10, no. 1, pp. 381-393, 2013.

[12] A. June, "Research Paper on Optimized Utilization of Resources Using PSO and Improved Particle Swarm Optimization ( IPSO ) Algorithms in Cloud Computing," vol. 2, no. 2, pp. 499-505, 2014.

[13] Eberhart and Yuhui Shi, "Particle swarm optimization: developments, applications and resources," Proc. 2001 Congr. Evol. Comput. (IEEE Cat. No.01TH8546), vol. 1, no. February, pp. 81-86, 2015. 
[14] C. M. May, "Formal Methods for IT Security," pp. 1-44, 2007.

[15] A. Bertolino, "Software Testing Research: Achievements, Challenges, Dreams," Futur. Softw. Eng. (FOSE '07), no. September, pp. 85-103, 2007.
[16] I. Schieferdecker, J. Grossmann, and M. Schneider, "Model-Based Security Testing," Electron. Proc. Theor. Comput. Sci., vol. 80, no. Mbt, pp. 1-12, 2012.

[17] A. Bertolino, "Software Testing Research and Practice," Abstr. State Mach. 2003,pp. 1-21, 2003. 